\title{
Survival signal REG3 $\alpha$ prevents crypt apoptosis to control acute gastrointestinal graft-versus-host disease
}

\author{
Dongchang Zhao, ${ }^{1}$ Yeung-Hyen Kim, ${ }^{2}$ Seihwan Jeong, ${ }^{1}$ Joel K. Greenson, ${ }^{3}$ Mohammed S. Chaudhry, ${ }^{1}$ Matthias Hoepting, ${ }^{4}$ \\ Erik R. Anderson, ${ }^{1}$ Marcel R.M. van den Brink, ${ }^{5}$ Jonathan U. Peled, ${ }^{5}$ Antonio L.C. Gomes, ${ }^{5}$ Ann E. Slingerland, ${ }^{5}$ \\ Michael J. Donovan, ${ }^{6}$ Andrew C. Harris, ${ }^{7}$ John E. Levine, ${ }^{1}$ Umut Ozbek, ${ }^{1}$ Lora V. Hooper, ${ }^{8}$ Thaddeus S. Stappenbeck, ${ }^{9}$ \\ Aaron Ver Heul, ${ }^{9}$ Ta-Chiang Liu, ${ }^{9}$ Pavan Reddy, ${ }^{10}$ and James L.M. Ferrara ${ }^{1}$ \begin{abstract}
${ }^{3}$ Department of Pathology, University of Michigan, Ann Arbor, Michigan, USA. ${ }^{4}$ Department of Hematology, Hemostasis, Oncology and Stem Cell Transplantation, Hannover Medical School, Hannover, Germany. ${ }^{5}$ Memorial Sloan Kettering Cancer Center, Weill Cornell Medical College, New York, New York, USA. ${ }^{6}$ Department of Pathology, Icahn School of Medicine at Mount Sinai Hospital, New York, New York, USA. ${ }^{B}$ Blood and Marrow Transplantation Program, University of Utah, Salt Lake City, Utah, USA. ${ }^{8}$ Howard Hughes Medical Institute, Department of Immunology, University of Texas Southwestern Medical Center, Dallas, Texas, USA. 'Department of Pathology and Immunology, Washington University School of Medicine, St. Louis, Missouri, USA. ${ }^{10}$ Division of Hematology/Oncology,
\end{abstract} \\ 'The Tisch Cancer Institute and Division of Hematology/Medical Oncology, Icahn School of Medicine at Mount Sinai Hospital, New York, New York, USA. ${ }^{2}$ Department of Microbiology and Immunology, and \\ University of Michigan, Ann Arbor, Michigan, USA.
}

\begin{abstract}
Graft-versus-host disease (CVHD) in the gastrointestinal (GI) tract remains the major cause of morbidity and nonrelapse mortality after BM transplantation (BMT). The Paneth cell protein regenerating islet-derived $3 \alpha(\mathrm{REC} 3 \alpha)$ is a biomarker specific for GI CVHD. REG3 $\alpha$ serum levels rose in the systematic circulation as GVHD progressively destroyed Paneth cells and reduced GI epithelial barrier function. Paradoxically, CVHD suppressed intestinal REC3 $\gamma$ (the mouse homolog of human REC3 $\alpha$ ), and the absence of REG3 $\gamma$ in BMT recipients intensified GVHD but did not change the composition of the microbiome. IL-22 administration restored REC3 $\gamma$ production and prevented apoptosis of both intestinal stem cells (ISCs) and Paneth cells, but this protection was completely abrogated in Reg $3 \mathrm{~g}^{-/-}$mice. In vitro, addition of REG3 $\alpha$ reduced the apoptosis of colonic cell lines. Strategies that increase intestinal REC3 $\alpha / \gamma$ to promote crypt regeneration may offer a novel, nonimmunosuppressive approach for GVHD and perhaps for other diseases involving the ISC niche, such as inflammatory bowel disease.
\end{abstract}

\section{Introduction}

Acute graft-versus-host disease (GVHD) is the most serious complication of allogeneic BM transplantation (BMT) and was noted in animal models more than 6 decades ago $(1,2)$. Acute GVHD predominantly involves the skin, liver, and lower gastrointestinal (GI) tract (3).

Without rigorous depletion of donor T cells from the graft, this complication occurs in 30\%-70\% of patients after allogeneic BMT (4-6). Standard treatment of acute GVHD is the administration of systemic corticosteroids and additional immunosuppressive agents; only half of patients who develop acute GVHD will have a complete response to corticosteroid therapy, although the numbers vary widely between studies (7). The outcome for patients with severe GVHD of the lower GI tract is poor, with $25 \%$ overall survival (8). We undertook this study to understand the biologic role of regenerating islet-derived $3 \alpha / \gamma(\mathrm{REG} 3 \alpha / \gamma)$ in GI GVHD.

Authorship note: DZ and YHK contributed equally to this work. Conflict of interest: The authors have declared that no conflict of interest exists. Submitted: December 14, 2017; Accepted: August 7, 2018. Reference information: J Clin Invest. 2018;128(11):4970-4979. https://doi.org/10.1172/JCI99261.

\section{Results}

An elevated REG3 $\alpha$ blood level is a strong indicator of GI GVHD that drives transplant reported mortality (9-12). We observed that increased blood levels of REG3 $\alpha$ in patients with GVHD inversely correlated with the number of Paneth cells (Figure 1, A-C) and observed a similar inverse correlation in 2 mouse models of GVHD (Figure 1, D-I, and Supplemental Figure 1; supplemental material available online with this article; https://oi.org/10.1172/ JCI99261DS1). This inverse correlation continued over time and increased with GVHD severity independently of BMT conditioning (Figure 2). IL-22 induces REG3 $\gamma$ (13-15), and the production of IL-22 decreased very rapidly during GVHD (Figure 2). Increased $R e g 3 g$ expression was not observed in any other tissue, including lung, liver, and pancreas (data not shown), suggesting that damage to the GI crypt resulted in the loss of REG3 $\gamma$ into the systemic circulation. REG3 $\alpha$ serum levels remained elevated, often by an order of magnitude, after 1 week of systemic steroid therapy in patients with GI GVHD and predicted increased nonrelapse mortality, confirming the significance of continued serum elevations with respect to long-term clinical outcomes (Figure 3 and ref. 16).

We next investigated the role of REG3 $\gamma$ in GVHD using REG3 $\gamma$-deficient (Reg3 $g^{-/-}$) mice as BMT recipients. Reg3 $g^{-/-}$mice experienced more severe clinical GVHD and greater mortality 
A

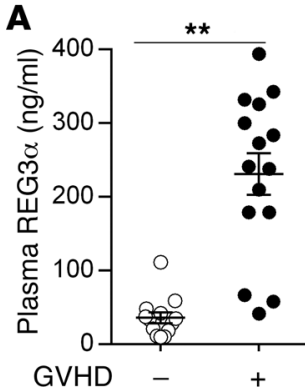

D

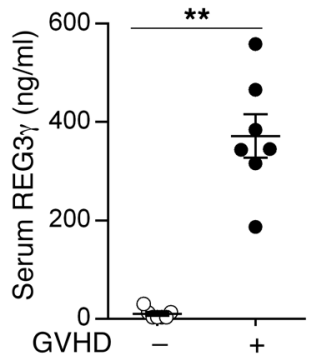

G

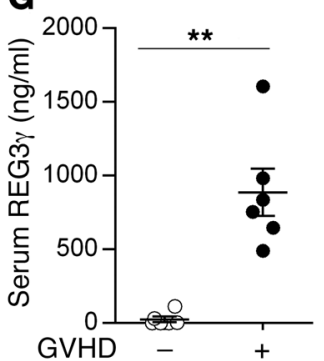

B

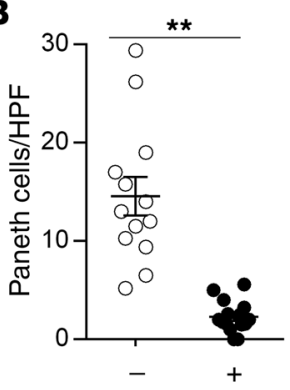

$\mathbf{E}$

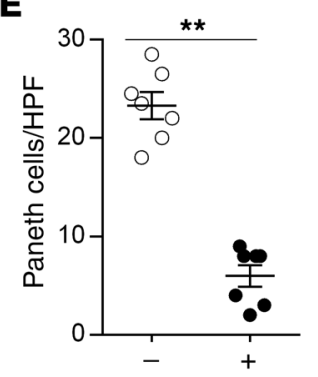

H

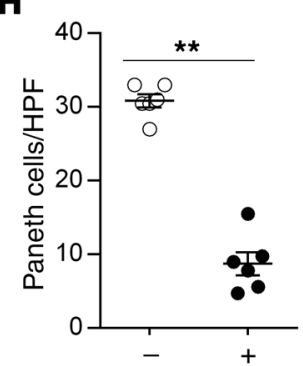

C

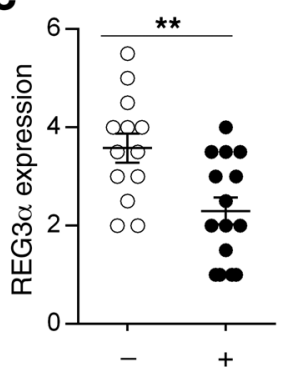

$\mathbf{F}$

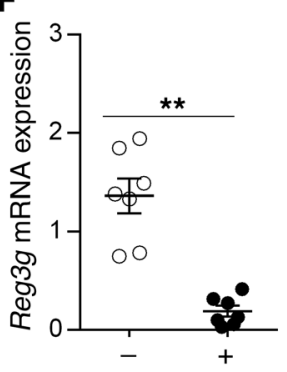

I

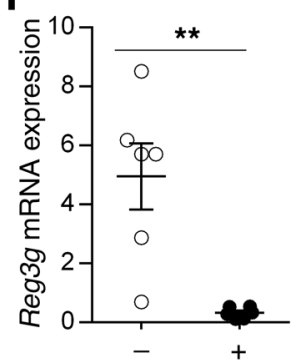

Figure 1. REC3 $\alpha / \gamma$ levels in the plasma/serum and intestinal mucosa during GVHD. (A-C) Samples were collected from 28 allogeneic BMT patients without GVHD (white circles, $n=13$ ) and with GVHD (black circles, $n=15$ ). (A) Plasma concentrations of REC3 $\alpha$ measured by ELISA. (B) Average Paneth cell numbers per high-power field (HPF) in the same biopsies. (C) Semiquantitative REG3 $\alpha$ expression in the duodenum. (D-F) B6 mice underwent BMT from syngeneic B6-Ly5.1 donors (GVHD -, white circles, $n=7$ ) or allogeneic C3H.SW donors (GVHD +, black circles, $n=7$ ), and samples were analyzed on day +7 after BMT. (D) Serum REC3 $\gamma$ levels measured by ELISA. (E) Average Paneth cell numbers per HPF in ileal tissue from the same mice. (F) Ileal tissue Reg $3 g$ mRNA expression measured by quantitative PCR (qPCR). (G-I) B6D2F1 mice underwent BMT from syngeneic B6D2F1 donors (CVHD -, white circles, $n=6$ ) or allogeneic B6 donors (CVHD +, black circles, $n=6$ ), and samples were analyzed as before on day +7 after BMT. (G) Serum REG3 $\gamma$ levels. (H) Average Paneth cell numbers per HPF in ileal tissue. (I) lleal tissue Reg3g mRNA expression. ${ }^{*} P<0.01$, unpaired 2 -tailed $t$ test. Data are expressed as mean \pm SEM. than WT mice in 2 GVHD models (Figure 4, A-C). The histologic severity of GVHD, as measured by Paneth cell apoptosis and loss, also increased in Reg3g $g^{-/-}$BMT recipients; so did the number of crypt cells expressing cleaved caspase-3, a hallmark of apoptosis (Figure 4, D-F, and Supplemental Figures 2 and 3). Reg3 $g^{-/-}$allogeneic BMT recipients also exhibited significantly more $\mathrm{CD} 8^{+} \mathrm{IFN}-\gamma^{+}$ effector $\mathrm{T}$ cells in the lamina propria as well as increased hepatic infiltrates, confirming greater histologic damage in the absence of recipient REG3 $\gamma$ (Supplemental Figures 3 and 4). The composition of fecal microbial communities did not differ significantly, however, between WT and Reg3 $g^{-/-}$BMT recipients either before transplant or during GVHD (Figure 4, G-I, and Supplemental Figure 5).

Although IL-22 has pleiotropic effects and can increase GVHD severity in some models $(17,18)$, prophylactic administration of IL-22 prevented GVHD and increased REG3 $\gamma$ production in the ileum as previously reported (15) while simultaneously decreasing serum REG3 $\gamma$ levels (Figure 5 and Supplemental Figure 6). IL-22 treatment of mice also reversed established GVHD in 2 separate models, and decreased release of REG3 $\gamma$ into serum (Figure 6 and Supplemental Figure 7). IL-22 was completely unable, however, to prevent clinical and histologic GVHD and Paneth cell loss in Reg $3 g^{-/-}$recipients in 2 separate models, confirming the need for REG3 $\gamma$ to repair the integrity of GI epithelium (Figure 7 and Supplemental Figures 8-10). mRNA expression of other antimicrobial Paneth cell proteins that are disrupted during GVHD (19), such as
Defa1, Defa2, S100a8, and S100a9, did not consistently change in Reg $3 g^{-/-}$recipients (Supplemental Figure 11).

To investigate the effect of REG3 $\gamma$ on intestinal stem cells (ISCs), we bred $R e g 3 g^{-1-}$ mice with mice heterozygous for green fluorescent protein (GFP) in Lgr5 ${ }^{+}$enterocytes (20) to use as BMT recipients. We confirmed that the loss of ISCs during GVHD (21) was not reversed by IL-22 treatment in $\mathrm{Reg} 3 \mathrm{~g}^{-/-}$mice (Figure 8A and Supplemental Figure 12). We next quantified ISC apoptosis and proliferation using established flow cytometry protocols (22). Surprisingly, IL-22 did not affect ISC proliferation under any conditions, but reversed apoptosis only in WT and not in Reg3 $3 g^{-/-}$recipients; the number of ISCs correlated directly with Reg3g mRNA expression (Figure 8, B-D, and Supplemental Figures 13 and 14). We then directly evaluated the antiapoptotic properties of REG3 $\alpha$ in intestinal epithelium in vitro. Addition of REG3 $\alpha$ in vitro to colonic epithelial cell lines undergoing apoptotic stress reduced the cleavage of caspase-3, caspase-8, and PARP and increased cell viability in a dose-dependent manner, confirming its role as a survival factor for epithelial cells (Figure 8, E-G, and Supplemental Figure 15).

\section{Discussion}

We report here that the production of REG $3 \alpha / \gamma$ in the GI tract falls as acute GVHD progresses and inversely correlates with its concentration in the serum, supporting the concept of this biomarker as a "liquid biopsy" that quantifies crypt damage and the loss of 

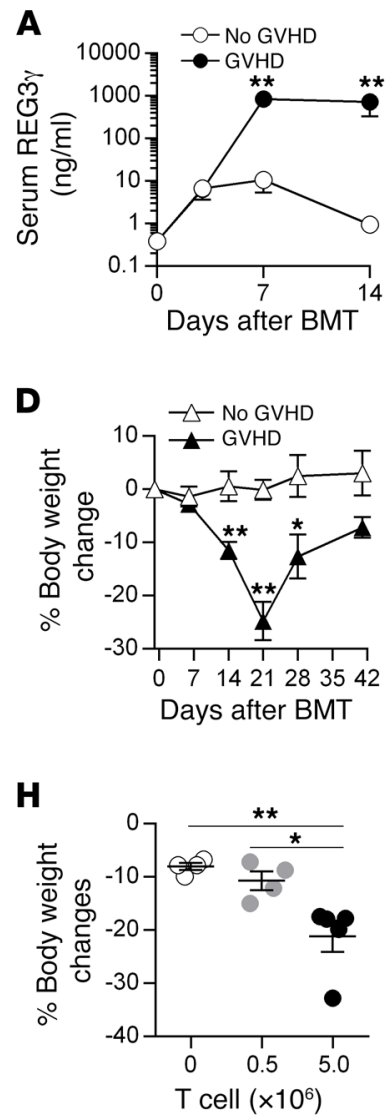
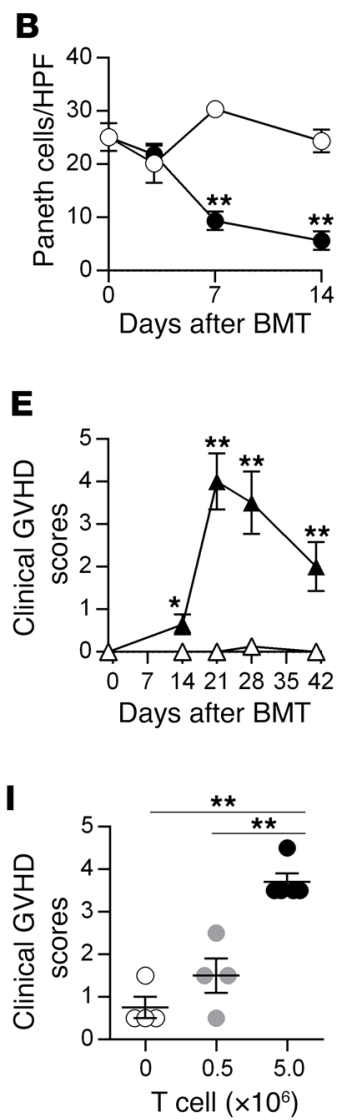
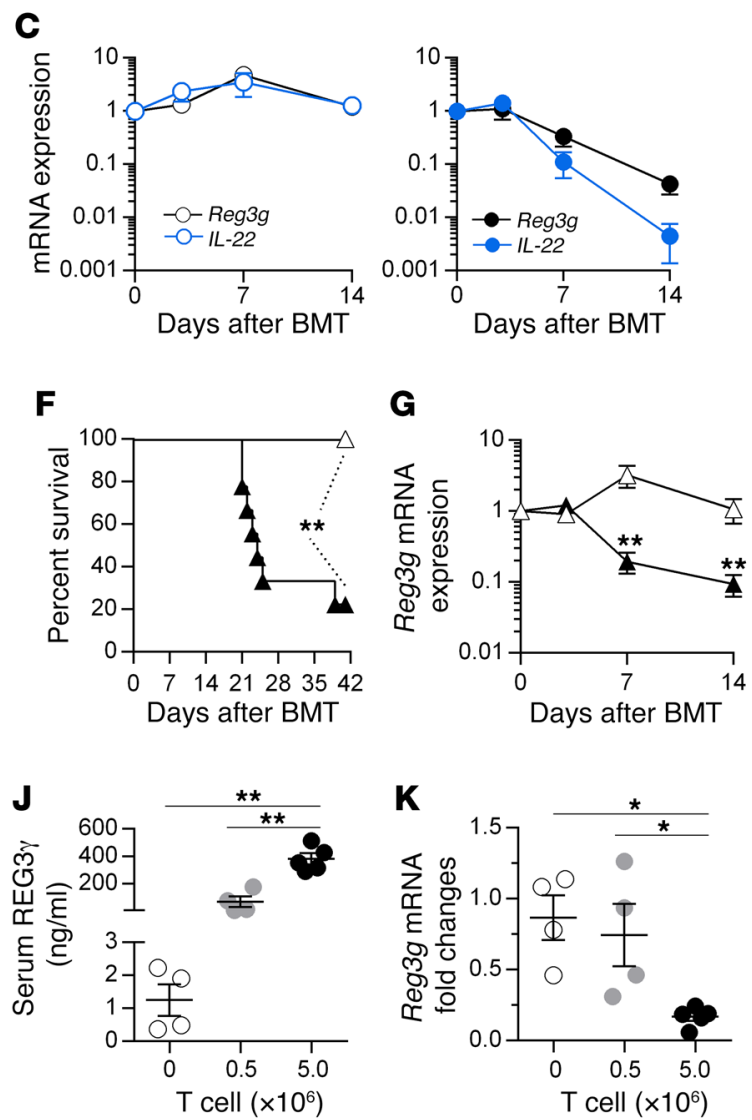

Figure 2. Time course of REG3 $\gamma$ and IL-22 expression during GVHD. (A-C) B6D2F1 mice underwent BMT from syngeneic B6D2F1 donors (no GVHD, white circles) or allogeneic B6 donors (GVHD, black or blue circles), and samples were analyzed on days $+3,+7$, and +14 after BMT ( $n=5$ for each group on each day). (A) Serial REC3 $\gamma$ levels in the serum as measured by ELISA. (B) Serial average Paneth cell numbers per HPF in ileal tissue. (C) Serial Reg3g and IL-22 mRNA expression in the ileum was measured by qPCR. (D-C) B6D2F1 mice underwent BMT, without irradiation conditioning, from syngeneic B6D2F1 donors (no GVHD, white triangles) or allogeneic B6 donors (CVHD, black triangles). (D-F) Serial body weight measurement (D), clinical CVHD score (E), and survival (F) of mice after BMT. (G) Serial Reg3g mRNA expression in the ileum on days $+3,+7$, and +14 after BMT ( $n=6$ for each group on each day). ${ }^{*} P<0.05$, ${ }^{* *} P<0.01$, unpaired 2-tailed $t$ test $(\mathbf{A}-\mathbf{E}$ and $\mathbf{G})$; ${ }^{* *} P<0.01$, log-rank test $(\mathbf{F})$. (H-K) Following irradiation, B6 mice received $5.0 \times 10^{6} \mathrm{BM}$ cells plus $0 \times 10^{6}$ to $5.0 \times 10^{6} \mathrm{~T}$ cells, as indicated, from C3H.SW donors. Samples were analyzed on day +7 after BMT ( $n=4$ for each group). (H) Body weight measurement. (I) Clinical GVHD score. (J) Serum REG3 $\gamma$ levels. (K) Reg3g mRNA expression in the ileum. ${ }^{*} P<0.05,{ }^{* *} P<0.01,1$-way ANOVA. Data are expressed as mean \pm SEM.

ISCs and Paneth cells. Administration of IL-22 restores GI epithelial integrity and reduces GVHD because REG $3 \alpha / \gamma$ acts as a survival signal for ISCs and Paneth cells, preventing their apoptosis both in vitro and in vivo. The requirement of REG $3 \alpha / \gamma$ to repair damaged GI epithelium treatment indicates a previously unknown function for this protein in GI crypt homeostasis.

These results suggest the possibility of a more physiologic approach to the prevention and treatment of GI GVHD through restoration of the GI mucosal barrier rather than intensified systemic immunosuppression. Paneth cells, the "guardians of the crypt," secrete multiple antimicrobial peptides, including REG proteins, that help to generate the chemical/physical barrier of the GI mucosa (23). A disrupted microbiome constitutes a significant risk factor for both experimental and clinical $\operatorname{GVHD}(24,25)$ and might amplify GVHD by exposing enterocytes to Gram-positive pathogens (26). But analysis of the fecal microbiome demonstrated that the absence of REG3 $\gamma$ produced no significant difference in the microbial community architecture either before or during GVHD even though it caused greater crypt destruction and sig- nificantly increased the loss of ISCs and Paneth cells. This discrepancy suggested that the antimicrobial activity of REG3 $\gamma$ was not primarily responsible for its protective properties. Absence of host REG3 $\gamma$ thus amplified GVHD by accelerating apoptosis of the crypts, allowing microbes to break the mucosal epithelial barrier and activate antigen-presenting cells that attracted and stimulated alloreactive donor T cells, furthering crypt damage (27).

Previous experiments with cultured pancreatic acinar cells and hepatocytes suggested that expression of REG3 $\beta$ could reduce the apoptosis mediated by TNF- $\alpha$, an important mediator of $\operatorname{GVHD}(28,29)$. Our results directly demonstrate a survival function for REG $3 \alpha / \gamma$ that is key to restoration of epithelial integrity during GVHD. A recently published approach to GVHD prevention is the promotion of Paneth cell development from ISCs through the administration of R-spondin-1, a Wnt agonist (30). It is unknown whether R-spondin-1 requires REG3 $\gamma$ to increase Paneth cell number as does IL-22. In this regard it is interesting to note that although IL-22 increases ISC number in vivo and stimulates organoid growth in vitro, it does not act as a mito- 
A

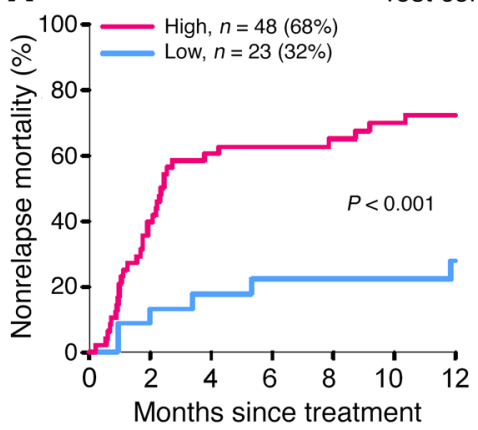

Test cohort $(n=71)$

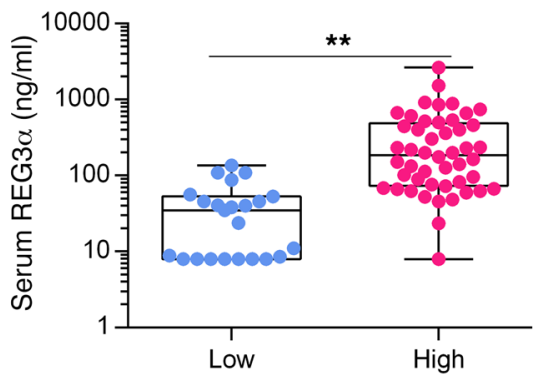

Figure 3. Serum REG3 $\alpha$ levels and correlates with nonrelapse mortality in patients with GI GVHD. Patients with GI GVHD ( $n=145)$ who were treated with systemic steroids for at least 7 days provided blood samples for biomarker analysis and were divided according to biomarker probabilities into low (blue) and high (pink) groups as previously published (16). Twelve-month nonrelapse mortality and REG3 $\alpha$ serum levels are shown for test $(n=71)(\mathbf{A})$ and validation $(n=74)\left(\right.$ B) cohorts. ${ }^{* *} P<0.01$, unpaired 2-tailed $t$ test. Data are expressed as box-andwhisker plots.
B

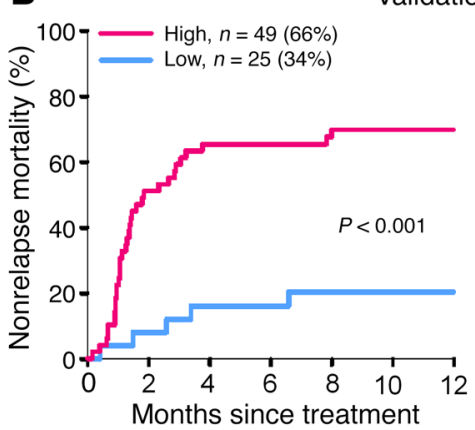

Validation cohort $(n=74)$

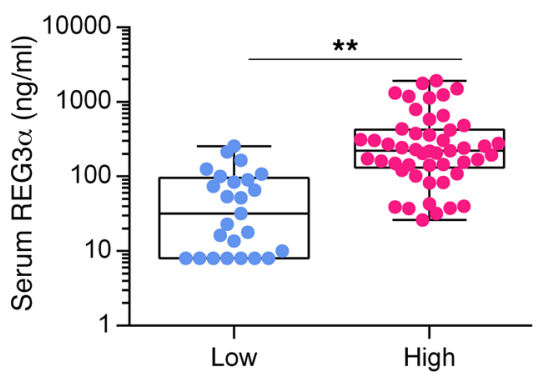

gen as was initially thought; rather it prevents the apoptosis (or necroptosis) caused by the inflammation of GVHD through the induction of REG3 $\gamma$. Thus, in addition to its well-defined antimicrobial properties, REG3 $\gamma$ provides critical survival signals to cells in the stem cell niche.

The potential limitations of this study should be noted. First, the mechanistic insights derive from mouse models and cell lines that cannot reproduce the complexity of human disease. Despite many tight correlations between human and murine data, some experiments do not and cannot have precise human equivalents, e.g., quantification of ISCs through a green fluorescent protein. Another important limitation relates to differences along the axial geography of the bowel. Both the large and the small intestine contain Lgr5 $5^{+}$ISCs, but Paneth cells are largely absent in the colon, where the stem cell niche is supported by deep secretory cells that possess some similarities to Paneth cells (31). Experiments are currently in progress to determine whether REG3 $\gamma$ also acts as a survival signal for colonic ISCs in vivo. If restoration of the survival signal provided by REG3 $\gamma$ can repair damaged intestinal epithelium of both small and large intestines, this approach may offer a novel, nonimmunosuppressive strategy not only for GVHD but for other immunologically mediated diseases of the bowel.

\section{Methods}

Patients. Patients were studied with the approval of the Institutional Review Boards at the University of Michigan, and all patients gave written informed consent in accordance with the Declaration of Helsinki. Twenty-eight patients, who consented to a prospective observational trial of allogeneic BMT complications and underwent allogeneic BMT from 2002 to 2007, were available for analysis; samples were obtained from patients who developed GI symptoms and had a duodenal biopsy and plasma collection at the time of onset of symptoms. All patients without GVHD had a histologic grade of 0 , and the median time of biopsy was day +37 (range 15-78); all those with GVHD had a histologic grade of 3, and the median time of biopsy was day +65 (range 13-138).

Mice. C57BL/6 (B6; H-2 $\left.{ }^{\mathrm{b}}, \mathrm{CD} 45.2^{+}\right)$, B6-Ly5.1 (H-2 $\left.2^{\mathrm{b}}, \mathrm{CD} 45.1^{+}\right), \mathrm{B} 6$ $\times$ DBA2 $\mathrm{F}_{1}\left(\mathrm{~B} 6 \mathrm{D} 2 \mathrm{~F} 1 ; \mathrm{H}-2^{\mathrm{b} / \mathrm{d}}\right)$, and BALB/c $\left(\mathrm{H}-2^{\mathrm{d}}, \mathrm{CD} 90.2^{+}\right)$mice were purchased from Charles River Laboratories. C3H.SW (H-2, CD 45.2 $\left.2^{+}\right)$ mice were purchased from The Jackson Laboratory. Reg $3 g^{-1-}\left(\mathrm{H}-2^{\mathrm{b}}\right)$ mice were obtained from Lora V. Hooper (26). B6-Lgr5-EGFP-IREScreERT2 (Lgr5-EGFP') mice were a gift from Linda C. Samuelson (University of Michigan, Ann Arbor, Michigan, USA). $R e g 3 g^{-/-}$mice were bred with WT Lgr5-EGFP ${ }^{+}$mice to generate dual Reg3g-/-/Lgr5-EGFP ${ }^{+}$ mice (B6 background). For BMT experiments, WT and $R e g 3 g^{-/}$recipient mice were cohoused for a minimum of 2 weeks, in order to reduce heterogeneity in the microbiota between the strains. All animals were cared for under the regulations reviewed and approved by the University Committee on the Use and Care of Animals, per University Laboratory Animal Medicine guidelines at the University of Michigan or the Icahn School of Medicine at Mount Sinai.

$B M T$. BMT was performed as previously described $(32,33)$. Briefly, BM cells were collected from the femurs and tibiae of euthanized donor mice. Donor T cells were obtained from the spleens of donor mice by positive selection using CD90.2 magnetic beads (Miltenyi Biotec), according to the manufacturer's instructions. Unless otherwise stated, B6D2F1 recipients were given a total of 1,250 cGy totalbody irradiation, split into 2 doses separated by 4 hours on day -1 , and then injected with $2 \times 10^{6}$ to $4 \times 10^{6}$ splenic T cells and $5 \times 10^{6} \mathrm{BM}$ cells from allogeneic B6 donors on day 0. B6 recipients were irradiated with 1,100 cGy total-body irradiation, as a single dose on day -1 , and injected on day 0 with $50 \times 10^{6}$ whole splenic cells and $5 \times$ $10^{6} \mathrm{BM}$ cells from MHC-matched, minor antigen-disparate $\mathrm{C} 3 \mathrm{H}$.SW donors or $5 \times 10^{6}$ whole splenic cells and $5 \times 10^{6} \mathrm{BM}$ cells from MHCmismatched BALB/c donors. 
A

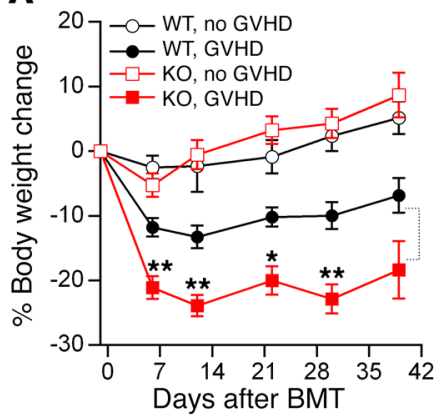

D
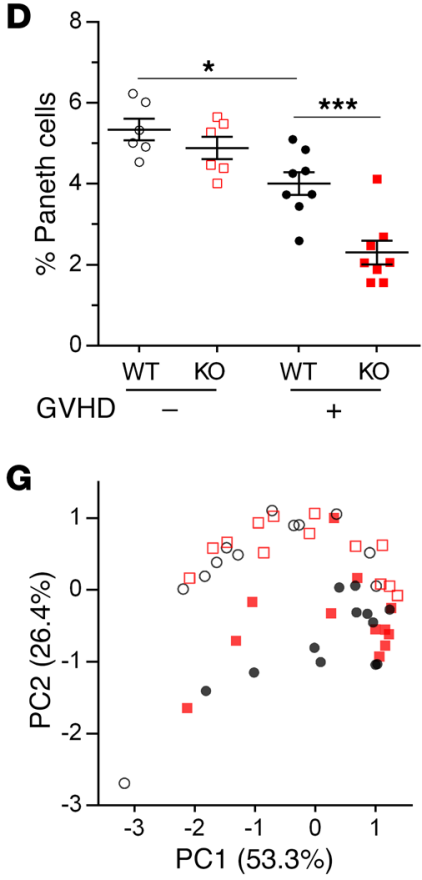

B

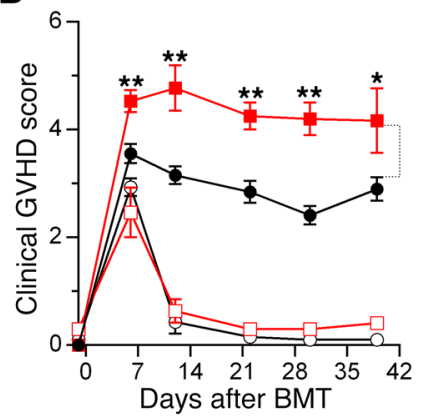

c

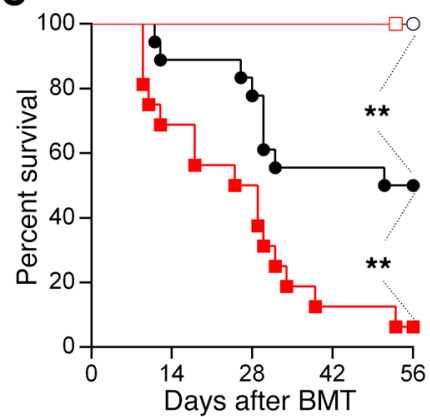

E
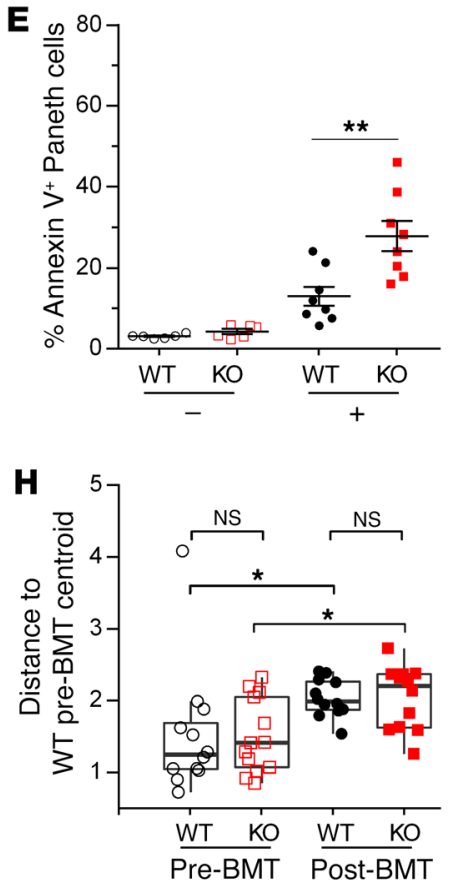

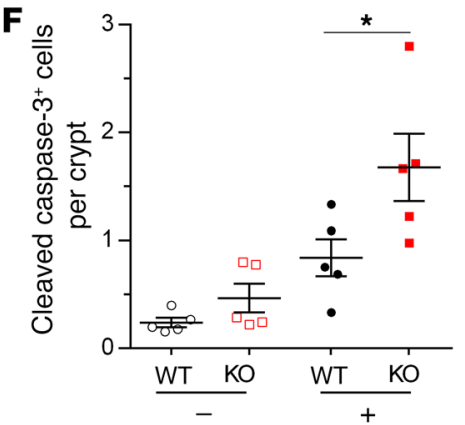

I

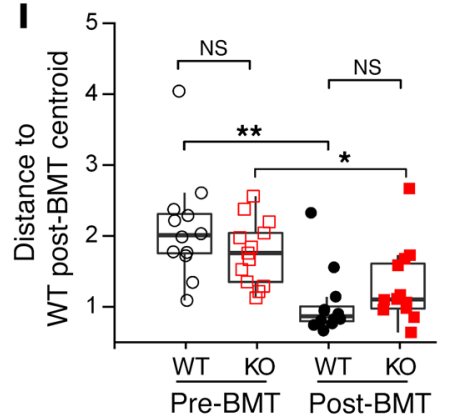

Figure 4. REG3 $\gamma$ absence increases GVHD severity. WT B6 and B6-Reg3g-/- (KO) mice underwent BMT from B6-Ly5.1 donors (GVHD -: WT, white circles; KO, white squares) or C3H.SW donors (CVHD +: WT, black circles; KO, red squares). (A-C) Serial body weight measurement (A), clinical CVHD score (B), and survival (C) of mice after BMT (WT, white circles, $n=7$; KO, white squares, $n=7$; WT, black circles, $n=18$; KO, red squares, $n=16$ ). (D-F) On day +7 after BMT, WT or KO recipients (Lgr5-EGFP+) were euthanized. Small intestine crypt cells were isolated and analyzed by flow cytometry. Small intestine sections were stained by immunochemistry. ( $\mathbf{D}$ and $\mathbf{E}$ ) Quantification of Paneth cells (D) and quantification of Paneth cells undergoing early apoptosis by annexin $\mathrm{V}^{+}$staining (E) (WT, white circles, $n=6$; KO, white squares, $n=6$; WT, black circles, $n=8$; KO, red squares, $n=8$ ). (F) Quantification of cleaved caspase- $3^{+}$cells per crypt of ileum sections in recipients (each group, $n=5$ ). ${ }^{*} P<0.05,{ }^{* *} P<0.01,{ }^{* * *} P<0.001$, unpaired 2-tailed $t$ test $(\mathbf{A}, \mathbf{B}$, and $\mathbf{D}-\mathbf{F}) ;{ }^{* *} P<0.01$, log-rank test (C). Data are expressed as mean \pm SEM. (G-I) Microbiome composition of fecal samples from WT and KO mice 1 day before BMT (Pre-BMT) (WT, white circles, $n=12$; KO, white squares, $n=13$ ) or day +7 after BMT (Post-BMT) (WT, black circles, $n=12$; KO, red squares, $n=13$ ). (C) Principal component analysis was computed from the Bray-Curtis $\beta$-diversity matrix among all samples. ( $H$ and $\mathbf{I})$ Bray-Curtis compositional distances were measured from the centroid of WT pre-BMT samples (H) or post-BMT samples (I). NS, $P>0.05$; ${ }^{*} P<0.05$, ${ }^{*} P<0.01$, Wilcox test.

In all experiments, transplantation with cells from the appropriate syngeneic donor or BM only was used as a control. Donor and recipient mice were sex-matched and were 8-16 weeks of age at the time of transplantation. Survival was monitored daily, and clinical GVHD was assessed weekly by a previously described scoring system (34).

Administration of IL-22 in vivo. Recombinant mouse IL-22 (carrierfree) was purchased from BioLegend. For prophylaxis experiments, mice received i.p. injections of $10 \mu$ g of IL-22 in PBS, or PBS as a control, on alternate days from day +1 after BMT. Mice were given either 3 doses (when GI tissues were analyzed on day +7 ) or 6 doses (for longerterm assessment of GVHD scores and survival).
To investigate the effect of treatment with IL-22 after development of GVHD, mice received i.p. injections of $10 \mu \mathrm{g}$ of IL-22 in PBS, or PBS as a control, starting on day +7 after BMT. Mice were given either 3 daily doses (when GI tissues were analyzed on day +10 ) or a total of 7 total doses on days 7, 8, 9, 11,13, 15, and 17 (for longer-term GVHD studies).

All animals were individually ear-tagged before BMT ( 4 animals per cage). At the time of randomization to receive IL-22 or PBS alone (day 0 for prophylaxis experiments and day 7 for treatment experiments), the first 2 mice were assigned to the IL-22 group and the second 2 mice were assigned to the PBS group. For experiments evaluating treatment beginning on day 7, animals with severe GVHD (weight loss of more than 
A

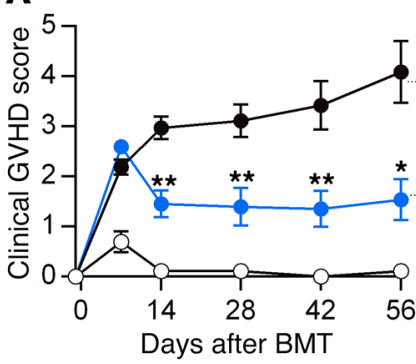

B

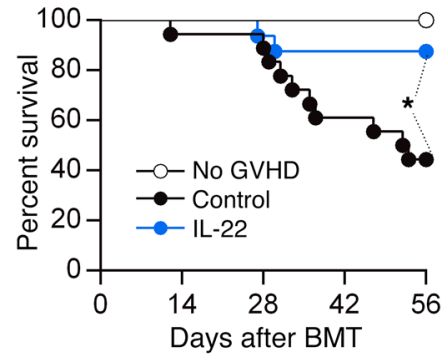

C

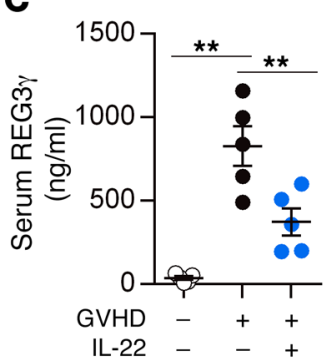

D

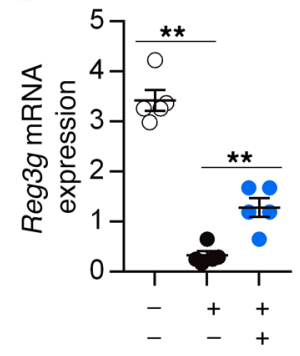

E

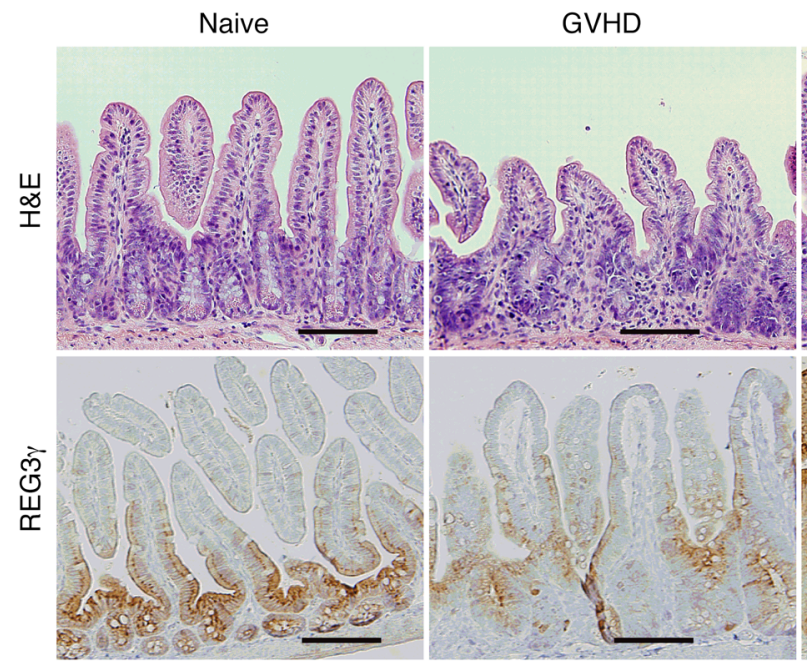

GVHD + IL-22

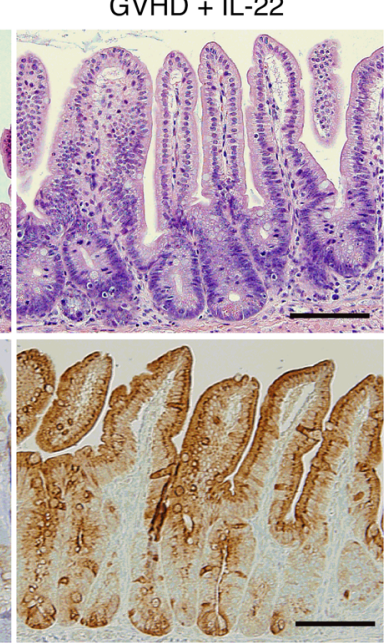

Figure 5. Prophylactic administration of IL-22 reduces GVHD. B6D2F1 mice received PBS or IL-22 injections from day +1 after BMT from B6 donors (GVHD, + ) or B6D2F1 donors (No GVHD, -). (A and B) Clinical GVHD score (A) and survival (B) of mice after BMT (GVHD +: PBS, black circles, $n=18$; IL-22, blue circles, $n$ $=16$; GVHD -, white circles, $n=7$ ). (C-E) Samples were analyzed on day +7 after BMT. (C) Serum REC3 $\gamma$ levels. (D) lleal tissue Reg3g mRNA expression. ${ }^{*} P<0.05$, ${ }^{* *} P<0.01$, unpaired 2-tailed $t$ test (A), 1-way ANOVA (C and D); ${ }^{*} P<0.05$, log-rank test (B). (E) H\&E (top) staining and REC3 $\gamma($ bottom) staining by immunohistochemistry in ileum from individual mice with or without IL-22 treatment. Naive B6D2F1 mice were used as controls. Images were taken with an Olympus BX51 with a $\times 20$ objective. Scale bars: $100 \mu \mathrm{m}$. Data are expressed as mean \pm SEM.

25\%) were excluded before randomization. This criterion was established before BMT and the subsequent development of disease.

5-Ethynyl-2'-deoxyuridine incorporation in vivo assay. ISC proliferation was determined using 5-ethynyl-2'-deoxyuridine (EdU) incorporation into DNA. Lgr5-EGFP ${ }^{+}$mice were injected i.p. with 200 $\mu \mathrm{l}$ of $5 \mathrm{mM}$ EdU solution (Invitrogen) in PBS 2 hours before smallintestinal samples were collected. EdU was detected with the Click-iT Plus EdU Flow Cytometry Assay kit according to the manufacturer's instructions (Invitrogen).

Histopathology and immunohistochemistry. For pathologic analyses, samples from patients (duodenal biopsies) and mice (distal ilea and livers) were fixed in 10\% neutral-buffered formalin, embedded in paraffin, sectioned, slide-mounted, and stained with H\&E (12). Paneth cells were identified by their eosinophilic granules by an experienced observer blinded to the treatment groups, in at least 3 high-power fields (HPFs) with an Olympus BX51 microscope. Using a highly reproducible technique (12), HPF was defined as a $\times 40$ objective with a field of $0.345 \mathrm{~mm}^{2}$. The counts from each HPF were averaged to give the number of Paneth cells per HPF.

Immunohistochemistry was performed with polyclonal rabbit antiREG3 $\alpha$ (Abcam, ab134309) at a 1:200 dilution for human samples and rabbit anti-REG3 $\gamma$ from the Lora V. Hooper laboratory (26) at a 1:8,000 dilution or rabbit anti-cleaved caspase-3 (clone 5A1E, Cell Signaling) at a 1:400 dilution for murine samples using a DAKO AutoStainer Link; slides were subsequently coated with a goat anti-rabbit IgG HRP conjugate (DAKO) at a 1:200 dilution and finally a diaminobenzidine (DAB) dilution to generate brown-colored signals. Slides were counterstained with Harris hematoxylin. Semiquantitative REG3 $\alpha$ expression from duodenum biopsies was scored on a 0-2 scale for 3 parameters: Paneth cells, epithelial cytoplasm, and stromal cells. Pictures from tissue sections were taken using a digital camera (DP70, Olympus) mounted on a microscope (BX51, Olympus) or an EVOS XL Core Imaging System from Life Technologies.

Quantitative PCR. Total RNA was isolated from the small intestine using the RNeasy RNA Isolation Kit (Qiagen) and was used to synthesize cDNA with the High-Capacity cDNA Reverse Transcription Kit (Applied Biosystems). Quantitative PCR analysis was performed using the SYBR Green Master Mix (Invitrogen) and specific primers. Signals were normalized to Gapdh levels within each sample, and normalized data were used to calculate relative levels of gene expression using $\Delta \Delta C_{t}$ analysis. Details of all primers used can be found in Supplemental Table 1 .

ELISA. REG3 $\alpha$ in human plasma was quantified with the REG3 $\alpha$ ELISA kit (MBL International, Japan) as described previously (11). REG3 $\gamma$ in mouse serum was measured by a REG3 $\gamma$ ELISA Kit (Cloud- 
A

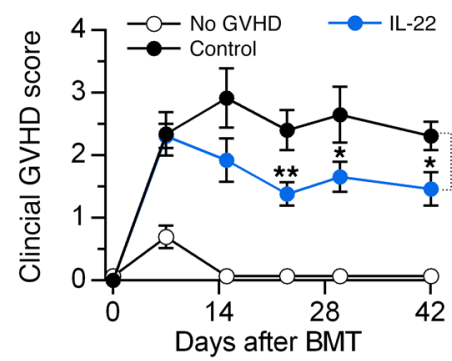

C

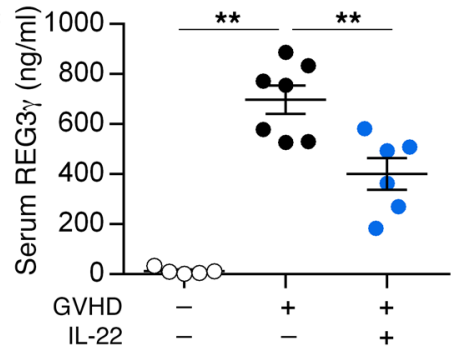

B

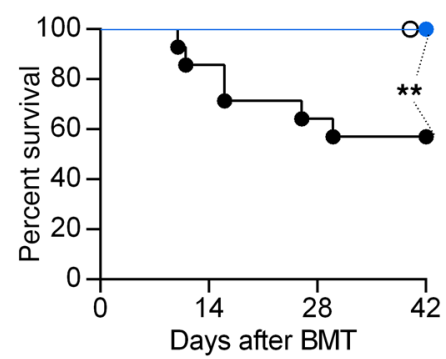

D

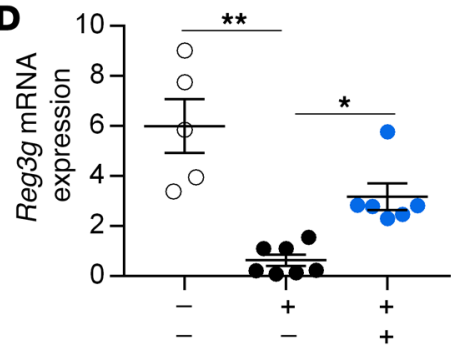

Figure 6. IL-22 treatment reverses GVHD. B6 mice received $\mathrm{BM}$ and T cells from $\mathrm{C} 3 \mathrm{H} . \mathrm{SW}$ donors (CVHD +) treated with PBS (black circles) or IL-22 (blue circles) from day +7 . As a control, mice received BM only (No GVHD, -, white circles). (A and $\mathbf{B}$ ) Clinical GVHD score (A) and survival (B) of mice after BMT (PBS, $n=14$; IL-22, $n=13$; no GVHD, $n=5$ ). Samples were analyzed on day +10 . (C) Serum REG3 $\gamma$ protein. (D) Small intestine tissue Reg3g mRNA. ${ }^{*} P<0.05$, ${ }^{* *} P<0.01$, unpaired 2-tailed $t$ test (A), 1-way ANOVA (C and $\mathbf{D}) ;{ }^{* *} P<$ 0.01 , log-rank test (B). Data are expressed as mean \pm SEM.
Clone Corp.) according to the manufacturer's protocol. Absorbance was measured with a SpectraMax M2 (Molecular Devices), and results were calculated with SoftMax Pro version 5.4 software (Molecular Devices).

Western blot. HT-29 cells were treated with human recombinant REG3 $\alpha$ protein (5940-RG-050, R\&D Systems) $(1,000 \mathrm{ng} / \mathrm{ml})$ followed by treatment with birinapant $(10 \mathrm{nM})$ and recombinant human TNF- $\alpha$ (rhTNF- $\alpha ; 10 \mathrm{ng} / \mathrm{ml})$ for various time periods $(4,8$, and 16 hours). Cells were lysed in RIPA buffer containing protease and phosphatase inhibitors and sonicated with Bioruptor (Diagenode). Analysis was performed on total cell lysate. Protein was quan-
A

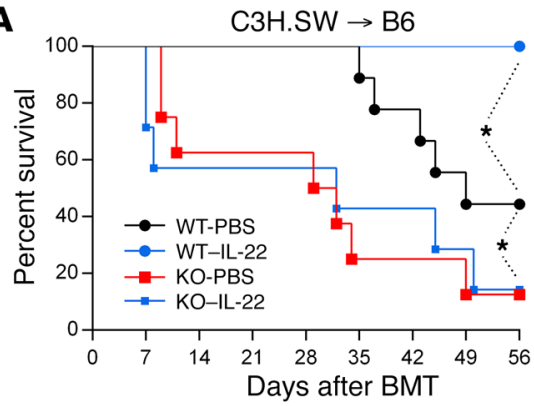

C

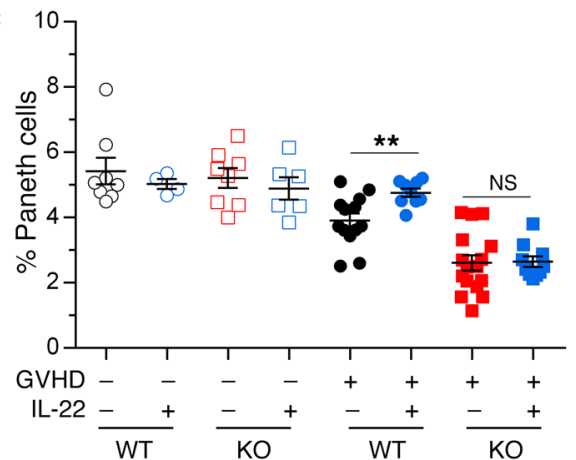

B
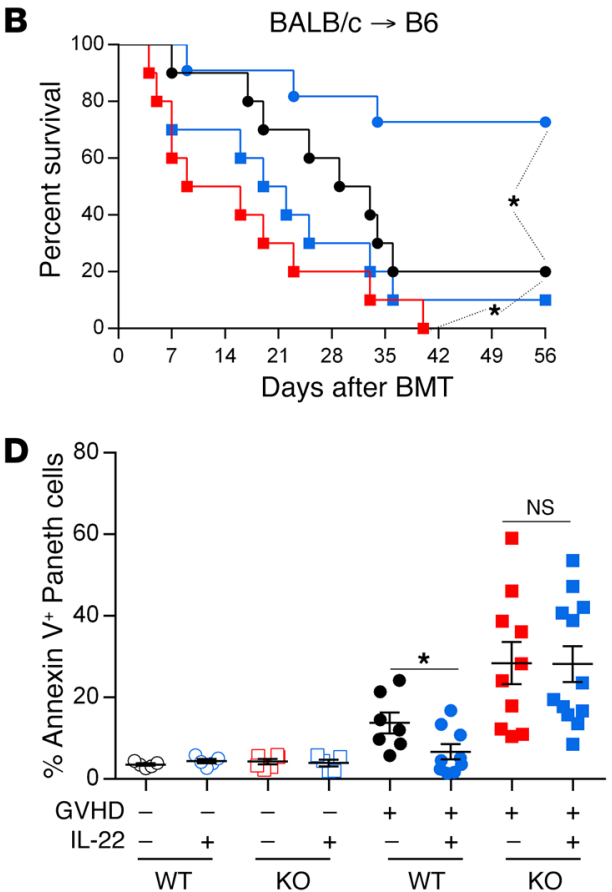

Figure 7. IL-22 requires REG3 $\gamma$ to reduce GVHD. (A) Survival of WT B6 and B6-Reg3g-/- (KO) mice received PBS or IL-22 injections from day +1 after BMT from C3H.SW donors (WT-PBS, black circles, $n=9$; WT-IL-22, blue circles, $n=8$; KO-PBS, red squares, $n=8$; KO-IL-22, blue squares, $n=7$ ). (B) Survival of WT B6 and B6-Reg3 $3 g^{-/-}$(KO) mice received PBS or IL-22 injections from day +1 after BMT from BALB/c donors (WT-PBS, black circles, $n=10 ;$ WT-IL-22, blue circles, $n=11$; KO-PBS, red squares, $n=10$; KO-IL-22, blue squares, $n=10) .{ }^{*} P<0.05$, log-rank test. (C and D) WT B6 and B6-Reg3g-1- (KO) mice (Lgr5-EGFP+) received PBS or IL-22 injections from day +1 after BMT from B6-Ly5.1 donors (GVHD -) or C3H.SW donors (CVHD +). On day +7 after BMT, small intestine crypt cells were analyzed by flow cytometry. (C) Quantification of Paneth cells (GVHD -: WT-PBS, empty black circles, $n=8$; WT-IL-22, empty blue circles, $n=4$; KO-PBS, empty red squares, $n=8$; KO-IL-22, empty blue squares, $n=6$; GVHD +: WT-PBS, filled black circles, $n=13$; WT-IL-22, filled blue circles, $n=9$; KO-PBS, filled red squares, $n=16$; KO-IL-22, filled blue squares, $n=10$ ). (D) Quantification of Paneth cells undergoing early apoptosis by annexin $\mathrm{V}^{+}$ staining (GVHD -: WT-PBS, empty black circles, $n=5$; WT-IL-22, empty blue circles, $n=5$; KO-PBS, empty red squares, $n=6$; KO-IL-22, empty blue squares, $n=5$; GVHD +: WT-PBS, filled black circles, $n=7$; WT-IL-22, filled blue circles, $n=9$; KO-PBS, filled red squares, $n=10$; KO-IL-22, filled blue squares, $n=12$ ). NS, $P>0.05 ;{ }^{*} P<0.05,{ }^{* *} P<0.01$, unpaired 2-tailed $t$ test. Data are expressed as mean \pm SEM. 
A

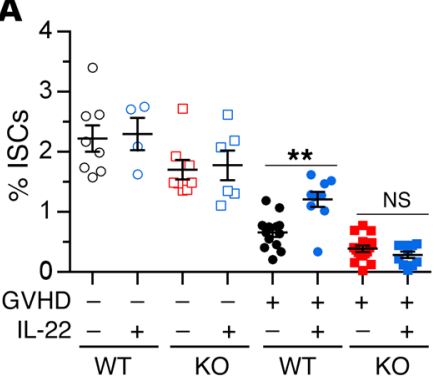

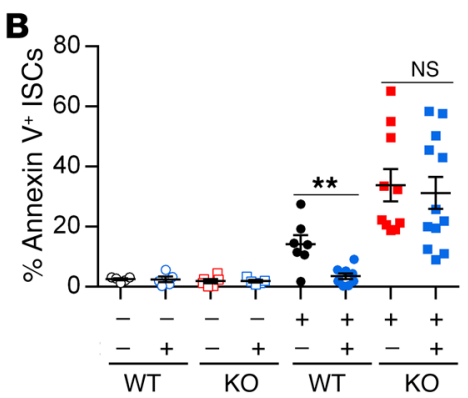
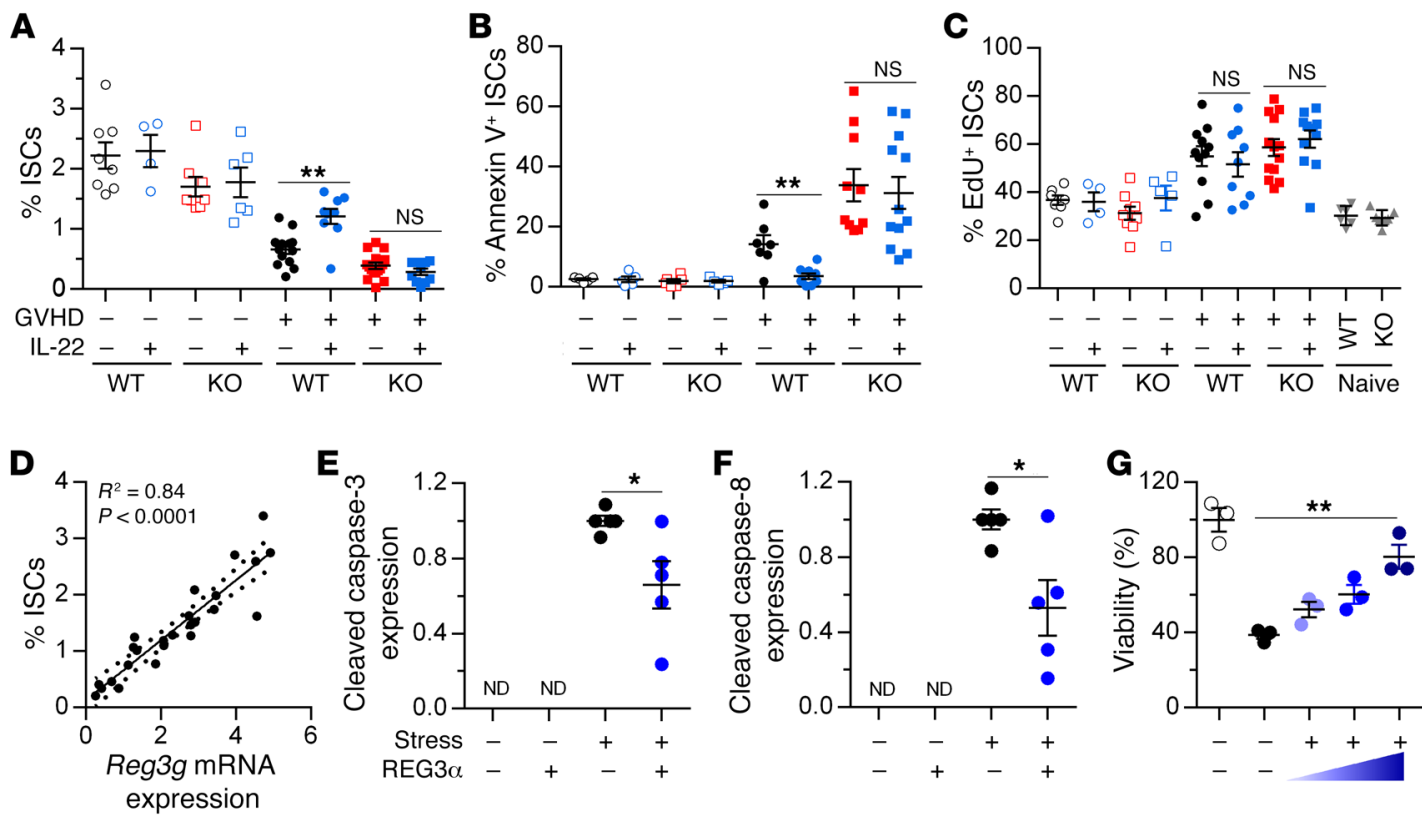

Figure 8. REG3 $\gamma$ protects ISCs from apoptosis. (A-D) WT B6 and B6-Reg $3 g^{-/-}(\mathrm{KO})$ mice (Lgr5-EGFP+) received PBS or IL-22 injections from day +1 after BMT from B6-Ly5.1 donors (CVHD -) or C3H.SW donors (CVHD +). On day +7 after BMT, small intestine crypt cells were analyzed by flow cytometry, and Reg3g mRNA was measured by qPCR. (A) Quantification of ISCs from mice in Figure 7. (B) Quantification of ISCs undergoing early apoptosis by annexin $\mathrm{V}^{+}$staining in the mice from Figure 7. Data are combined from 3 separate experiments. (C) Quantification of EdU+ ISCs in syngeneic recipients (GVHD -: WT-PBS, empty black circles, $n=7$; WT-IL-22, empty blue circles, $n=4$; KO-PBS, empty red squares, $n=9$; KO-IL-22, empty blue squares, $n=5$ ), allogeneic recipients (CVHD +: WT-PBS, filled black circles, $n=8$; WT-IL-22, filled blue circles, $n=9$; KO-PBS, filled red squares, $n=13$; KO-IL-22, filled blue squares, $n=11$ ), and naive WT (downward triangles, $n=5$ ) and KO (upward triangles, $n=5$ ) controls. (D) Correlation of ISCs and Reg3g mRNA expression in WT mice from 2 of the 3 experiments shown in A ( $n=25$ ), as calculated by the Pearson correlation coefficient. (E-C) HT-29 cells were cultured with recombinant REC3 $\alpha$ protein and apoptotic stimuli as described in Methods. Data are representative of 3 independent experiments. (E and $\mathbf{F}$ ) Cell lysates were collected after 16-hour cell culture for Western blot analysis. Quantification of cleaved caspase-3 (E) and cleaved caspase-8 (F) expression. (G) Quantification of cell viability by CellTiter-Clo 2.0 assay after 24 -hour cell culture. ${ }^{*} P<0.05,{ }^{* *} P<0.01$, unpaired 2-tailed $t$ test. Data are expressed as mean $\pm \mathrm{SEM}$. ND, not detectable.

tified using Pierce Rapid Gold BCA protein assay kit (Thermo Fisher Scientific). Thirty micrograms of lysate was loaded onto NuPAGE $4 \%-12 \%$ Bis-Tris protein gel or $12 \%$ Bis-Tris protein gel, and protein was separated by electrophoresis followed by transfer into nitrocellulose membrane. Membranes were blocked for 1 hour at room temperature with tris-buffered saline with tween (TBST) and 5\% nonfat milk and incubated overnight at $4^{\circ} \mathrm{C}$ with the following primary antibodies (Cell Signaling): PARP (9532S), cleaved PARP (5625S), caspase-8 (9746S), caspase-3 (29629S), cleaved caspase-3 (9661S), and $\beta$-actin (5125S). Proteins were detected with Pierce ECL Western Blotting Substrate (Thermo Fisher Scientific) or Lumigen ECL Ultra (Lumigen) following 1 hour of incubation at room temperature with anti-rabbit-HRP (7074S; Cell Signaling) or anti-mouse-HRP (7076S; Cell Signaling) antibody.

Apoptosis-induced colorectal epithelial cell viability measurement. 2.5 $\times 10^{5}$ cells of the colorectal cell line HT-29 were cultured in serumdeprived DMEM overnight. Human recombinant REG3 $\alpha$ protein (5940-RG-050, R\&D Systems) (10, 100, and 1,000 ng/ml) was added to culture for 2 hours, and then apoptosis was induced by addition of birinapant $(10 \mathrm{nM})$ and rhTNF- $\alpha(10 \mathrm{ng} / \mathrm{ml})$ to the culture for 24 hours. Cell viability was measured by CellTiter-Glo 2.0 assay kit (Promega) according to the manufacturer's protocol. Luminescence was measured with a SpectraMax M2 (Molecular Devices).

Lamina propria lymphocyte isolation. Lamina propria lymphocytes (LPLs) from small intestines were isolated as described previ- ously (26). Briefly, $5 \mathrm{~cm}$ distal ileum was opened longitudinally and rinsed in cold PBS, cut into 5-mm pieces, and washed twice with 2 mM EDTA (Thermo Fisher Scientific) and $1 \mathrm{mM}$ DTT (Thermo Fisher Scientific) in serum-free RPMI (MilliporeSigma) for 15 minutes at $37^{\circ} \mathrm{C}$ in a shaker at $150 \mathrm{rpm}$. Tissues were then washed with PBS supplemented with $3 \%$ FBS (MilliporeSigma) in a $100-\mu$ m cell strainer. Tissue was then cut into 1- to 3-mm pieces and incubated in a solution containing RPMI, 2\% FBS, 3.7 U Liberase (Roche Diagnostics), and 400 U DNase I (MilliporeSigma) for 15 minutes in an orbital shaker under the same conditions as above. Tissues were subsequently serially filtered through a $70-\mu \mathrm{m}$ and a $40-\mu \mathrm{m}$ cell strainer using ice-cold PBS, and LPLs were isolated after centrifugation on a Percoll gradient (GE Healthcare).

Intestinal crypt isolation and single-cell dissociation. Isolation of mouse intestinal crypts and dissociation of cells for analysis by flow cytometry were performed as previously described (35). Briefly, $5 \mathrm{~cm}$ proximal duodenum was opened longitudinally, rinsed in cold PBS, and cut into 5-mm pieces. To detach the crypts, small intestine pieces were incubated on ice in $10 \mathrm{mM}$ EDTA (Thermo Fisher Scientific) for 30 minutes. Intestine was then resuspended vigorously in PBS, leading to supernatant enriched in crypts, which was subsequently filtered through a $70-\mu \mathrm{m}$ cell strainer. Following centrifugation of isolated crypts, the pellet was disaggregated into single cells by incubation for 5 minutes with prewarmed $\left(37^{\circ} \mathrm{C}\right) 1 \times$ TrypLE Express (Gibco, Life Technologies) supplemented with $10 \mu \mathrm{M}$ ROCK inhibitor Y-27632 
dihydrochloride (Thermo Fisher Scientific) and $0.5 \mathrm{mM} \mathrm{N}$-acetylcysteine (MilliporeSigma).

Flow cytometry. After isolation, LPLs were further incubated with Cell Stimulation Cocktail (eBioscience) for 4 hours, and $1 \times 10^{6}$ cells were subsequently surface-stained with CD4, CD8, and TCR $\beta$ antibodies. For detection of polarized $\mathrm{T}$ cell subsets, cells were incubated overnight at $4^{\circ} \mathrm{C}$ in $400 \mu \mathrm{l}$ of Fix/Perm solution (BD Biosciences), and then stained in Perm/Wash buffer (BD Biosciences) with IFN- $\gamma$, IL-10, and IL-17A antibodies. For detection of Tregs, surface-stained LPLs were stained with FoxP3 antibody using the FoxP3/Transcription Factor Staining Buffer Set (eBioscience). Isolated, dissociated intestinal crypt cells were surface-stained with EpCAM, CD45, CD31, TER-119, and CD24 antibodies. Apoptotic intestinal crypt cells were detected using the Annexin V Apoptosis Detection Kit (eBioscience). DAPI, 7-aminoactinomycin D, and Fixable Live/Dead Cell Stain Kits (Invitrogen) were used for viability staining. All flow cytometry was performed on a FACSCanto or LSR Fortessa, using FACSDiva software (all BD Biosciences). Data were analyzed using FlowJo software (Tree Star). Full details of all antibodies used are in Supplemental Table 1.

$16 S$ sequencing. DNA from fecal pellets of individual mice were frozen until DNA was extracted by mechanical disruption (beadbeating) in phenol-chloroform. The V4-V5 region of the ribosomal RNA gene was amplified, sequenced, and computationally analyzed as described $(36,37)$.

Statistics. Statistical analysis was performed and graphs were generated using Prism software (GraphPad). Survival curves were plotted using Kaplan-Meier estimates and compared using the log-rank test. For survival experiments, a sample size of 7 per group provided $80 \%$ power to detect survival benefit of at least $40 \%$ with an $\alpha$ of 0.05 . For comparison of 2 groups, an unpaired 2-tailed $t$ test for parametric data was used. In the case of multiple comparisons, 1-way ANOVA with Bonferroni correction was used. Principal component analysis was per- formed using the R package, and Bray-Curtis compositional distances were compared using a Wilcoxon test. All data were tested for normality through application of the $F$ test with Prism software, and all tests were 2 -sided. Differences were considered significant when the $P$ value was less than 0.05. Data are always presented as mean and SEM for the different groups. Unless otherwise specified, all studies for which data are presented are representative of at least 2 independent experiments.

Study approval. Study of human blood samples was approved by the Icahn School of Medicine at Mount Sinai (IRB 15-0093). The mouse research project was approved by the Icahn School of Medicine at Mount Sinai (IACUC 2014-0202).

\section{Author contributions}

DZ, YHK, and JLMF designed the study. DZ, YHK, SJ, JKG, MSC, MH, ERA, MRMVDB, JUP, ALCG, AES, MJD, ACH, JEL, UO, TSS, AVH, TCL, and PR performed the experiments and analysis. $\mathrm{LVH}$ provided key reagents. DZ, MSC, and JLMF prepared the manuscript with input from all authors.

\section{Acknowledgments}

The authors thank the physicians and data management teams of the BMT programs at the University of Michigan and the University of Regensburg. This work was supported by NIH grants P01-CA039542 (to JLMF), 5T32AI078892 (to ERA), and R01CA228358 and R01-CA228308 (to MRMVDB), the American Cancer Society (CRP-13-306-06-COUN to JLMF), and Deutscher Akademischer Austauschdienst (to MH).

Address correspondence to: James L.M. Ferrara, Hess Center for Science and Medicine, Icahn School of Medicine at Mount Sinai, Room S6-110, 1470 Madison Avenue, New York, New York 10029, USA. Phone: 212.824.9365; Email: james.ferrara@mssm.edu.
1. Billingham RE. The biology of graft-versus-host reactions. Harvey Lect. 1966;62:21-78.

2. Gatti RA, Kersey JH, Yunis EJ, Good RA. Graftversus-host disease. Prog Clin Pathol. 1973;5:1-18.

3. Weisdorf DJ, et al. Acute upper gastrointestinal graft-versus-host disease: clinical significance and response to immunosuppressive therapy. Blood. 1990;76(3):624-629.

4. Przepiorka D, et al. Risk factors for acute graftversus-host disease after allogeneic blood stem cell transplantation. Blood.1999;94(4):1465-1470.

5 . Verneris MR, et al. HLA mismatch is associated with worse outcomes after unrelated donor reduced-intensity conditioning hematopoietic cell transplantation: an analysis from the Center for International Blood and Marrow Transplant Research. Biol Blood Marrow Transplant. 2015;21(10):1783-1789.

6. Pidala J, et al. Amino acid substitution at peptidebinding pockets of HLA class I molecules increases risk of severe acute GVHD and mortality. Blood. 2013;122(22):3651-3658.

7. Westin JR, et al. Steroid-refractory acute GVHD: predictors and outcomes. Adv Hematol. 2011;2011:601953.

8. Castilla-Llorente C, et al. Prognostic factors and outcomes of severe gastrointestinal GVHD after allogeneic hematopoietic cell transplantation.
Bone Marrow Transplant. 2014;49(7):966-971.

9. Hill GR, Ferrara JL. The primacy of the gastrointestinal tract as a target organ of acute graftversus-host disease: rationale for the use of cytokine shields in allogeneic bone marrow transplantation. Blood.2000;95(9):2754-2759.

10. MacMillan ML, DeFor TE, Weisdorf DJ. What predicts high risk acute graft-versus-host disease (GVHD) at onset?: identification of those at highest risk by a novel acute GVHD risk score. $\mathrm{Br} \mathrm{J}$ Haematol. 2012;157(6):732-741.

11. Ferrara JL, et al. Regenerating islet-derived $3-\alpha$ is a biomarker of gastrointestinal graft-versus-host disease. Blood. 2011;118(25):6702-6708.

12. Levine JE, et al. Low Paneth cell numbers at onset of gastrointestinal graft-versus-host disease identify patients at high risk for nonrelapse mortality. Blood. 2013;122(8):1505-1509.

13. Zheng Y, et al. Interleukin-22 mediates early host defense against attaching and effacing bacterial pathogens. Nat Med. 2008;14(3):282-289.

14. Sanos SL, et al. ROR $\gamma \mathrm{t}$ and commensal microflora are required for the differentiation of mucosal interleukin 22-producing NKp $46^{+}$cells. Nat Immunol. 2009;10(1):83-91.

15. Hanash AM, et al. Interleukin-22 protects intestinal stem cells from immune-mediated tissue damage and regulates sensitivity to graft versus host disease. Immunity. 2012;37(2):339-350. 16. Major-Monfried H, et al. MAGIC biomarkers predict long-term outcomes for steroid-resistant acute GVHD. Blood. 2018;131(25):2846-2855.

17. Couturier M, et al. IL-22 deficiency in donor T cells attenuates murine acute graft-versus-host disease mortality while sparing the graft-versus-leukemia effect. Leukemia. 2013;27(7):1527-1537.

18. Zhao K, et al. Interleukin-22 aggravates murine acute graft-versus-host disease by expanding effector $\mathrm{T}$ cell and reducing regulatory $\mathrm{T}$ cell. Jinterferon Cytokine Res. 2014;34(9):707-715.

19. Eriguchi Y, et al. Graft-versus-host disease disrupts intestinal microbial ecology by inhibiting Paneth cell production of $\alpha$-defensins. Blood. 2012;120(1):223-231.

20. Barker N, et al. Identification of stem cells in small intestine and colon by marker gene Lgr5. Nature. 2007;449(7165):1003-1007.

21. Lindemans CA, et al. Interleukin-22 promotes intestinal-stem-cell-mediated epithelial regeneration. Nature. 2015;528(7583):560-564.

22. Vermes I, Haanen C, Steffens-Nakken H, Reutelingsperger C. A novel assay for apoptosis. Flow cytometric detection of phosphatidylserine expression on early apoptotic cells using fluorescein labelled Annexin V. JImmunol Methods. 1995;184(1):39-51. 23. Ganz T. Paneth cells - guardians of the gut cell 
hatchery. Nat Immunol. 2000;1(2):99-100.

24. Jenq RR, et al. Regulation of intestinal inflammation by microbiota following allogeneic bone marrow transplantation. J Exp Med. 2012;209(5):903-911.

25 . Weber D, et al. Low urinary indoxyl sulfate levels early after transplantation reflect a disrupted microbiome and are associated with poor outcome. Blood. 2015;126(14):1723-1728.

26. Vaishnava S, et al. The antibacterial lectin RegIII $\gamma$ promotes the spatial segregation of microbiota and host in the intestine. Science. 2011;334(6053):255-258.

27. Zeiser R, Blazar BR. Acute graft-versus-host disease - biologic process, prevention, and therapy. N EnglJMed. 2017;377(22):2167-2179.

28. Malka D, et al. Tumor necrosis factor $\alpha$ triggers antiapoptotic mechanisms in rat pancreatic cells through pancreatitis-associated protein I activa- tion. Gastroenterology. 2000;119(3):816-828. 29. Simon MT, et al. HIP/PAP stimulates liver regeneration after partial hepatectomy and combines mitogenic and anti-apoptotic functions through the PKA signaling pathway. FASEB J. 2003;17(11):1441-1450.

30. Hayase E, et al. R-Spondin1 expands Paneth cells and prevents dysbiosis induced by graft-versushost disease. J Exp Med. 2017;214(12):3507-3518.

31. Sasaki N, et al. Reg $4^{+}$deep crypt secretory cells function as epithelial niche for $\mathrm{Lgr5}^{+}$ stem cells in colon. Proc Natl Acad Sci U S A. 2016;113(37):E5399-E5407.

32. Teshima T, et al. Acute graft-versus-host disease does not require alloantigen expression on host epithelium. Nat Med. 2002;8(6):575-581.

33. Reddy P, Maeda Y, Liu C, Krijanovski OI, Korngold R, Ferrara JL. A crucial role for antigenpresenting cells and alloantigen expression in graft-versus-leukemia responses. Nat Med. 2005;11(11):1244-1249.

34. Cooke KR, et al. LPS antagonism reduces graftversus-host disease and preserves graftversus-leukemia activity after experimental bone marrow transplantation. JClin Invest. 2001;107(12):1581-1589.

35. Sato T, et al. Paneth cells constitute the niche for Lgr5 stem cells in intestinal crypts. Nature. 2011;469(7330):415-418.

36. Staffas A, et al. Nutritional support from the intestinal microbiota improves hematopoietic reconstitution after bone marrow transplantation in mice. Cell Host Microbe. 2018;23(4):447-457.e4.

37. Shono Y, et al. Increased GVHD-related mortality with broad-spectrum antibiotic use after allogeneic hematopoietic stem cell transplantation in human patients and mice. Sci Transl Med. 2016;8(339):339ra71. 